\title{
Neuroaesthetics and beyond: new horizons in applying the science of the brain to the art of dance
}

\author{
Emily S. Cross • Luca F. Ticini
}

Published online: 5 January 2011

C The Author(s) 2011. This article is published with open access at Springerlink.com

\begin{abstract}
Throughout history, dance has maintained a critical presence across all human cultures, defying barriers of class, race, and status. How dance has synergistically co-evolved with humans has fueled a rich debate on the function of art and the essence of aesthetic experience, engaging numerous artists, historians, philosophers, and scientists. While dance shares many features with other art forms, one attribute unique to dance is that it is most commonly expressed with the human body. Because of this, social scientists and neuroscientists are turning to dance and dancers to help answer questions of how the brain coordinates the body to perform complex, precise, and beautiful movements. In the present paper, we discuss how recent advances in neuroscientific methods provide the tools to advance our understanding of not only the cerebral phenomena associated with dance learning and observation but also the neural underpinnings of aesthetic appreciation associated with watching dance. We suggest that future work within the fields of dance neuroscience and neuroaesthetics have the potential to provide mutual benefits to both the scientific and artistic communities.
\end{abstract}

Keywords Dance $\cdot$ Neuroscience $\cdot$ Neuroimaging $\cdot$ Neuroaesthetics

\footnotetext{
E. S. Cross $\cdot$ L. F. Ticini

Max Planck Institute for Human Cognitive and Brain Sciences, Stephanstraße 1a, 04103 Leipzig, Germany

E. S. Cross $(\bowtie)$

Department of Social and Cultural Psychology, Radboud Universiteit Nijmegen, P. O. Box 9104, 6500 HE Nijmegen, the Netherlands

e-mail: e.cross@psych.ru.nl

L. F. Ticini

Italian Society of Neuroesthetics "Semir Zeki", Florence, Italy

URL: www.neuroestetica.org
} 


\section{Introduction}

Across millennia, humans have expressed themselves through dance. Dance spans cultures, generations, social classes, and geography. Like music and the visual arts, dance is a universal human behavior whose earliest manifestations can be traced back to rituals and social expression (Boyer and Lienard 2006). ${ }^{1}$ While dance shares many features with other art forms, one attribute unique to dance is that its expression entails moving the body through time and space. ${ }^{2}$ To think about how we are able to coordinate our bodies to ascend a staircase, make a cup of tea, or ride a bicycle is impressive enough. To then imagine how the iconic modern dancer, José Limón, starting with the same raw materials of muscles, bones, and sinews, could use his body to leap, spin, contort, and move in sublimely beautiful ways, we begin to appreciate the vast chasm between our pedestrian, utilitarian, everyday actions, and the artistic, expressive, aesthetic actions that compose a dancer's movement repertoire.

While the study of dance performance and dance spectators' experience has traditionally been the realm of dance practitioners, scholars, and historians, more recently, psychologists and neuroscientists have been turning to dance to help address a number of questions about both the objective and subjective features of the relationship between perception and action. Two lines of inquiry are particularly ripe for research spanning dance and brain science disciplines. One of these concerns how dancers' brains can help to further illuminate the relationship between action experience and action observation. Another is how the observation of dance as an art form can inform our understanding of how the brain processes aesthetic experiences. In this paper, we begin by discussing what the nascent field of dance neuroscience has revealed about dance experience and observation at the neural level, and follow this by exploring how the brain sciences might be used to further probe aesthetic appreciation of dance as an art form. Finally, we conclude with a critical evaluation of how the dance and the brain sciences might continue along a path that is mutually informative and beneficial.

\section{Part 1: How dance helps to more fully characterize the relationship between perception and action in the human brain}

With the advent of cognitive psychology in the late nineteenth century, William James advanced the idea that action and perception in some manner converge or overlap (James 1890). Neurophysiological investigation into the brain's ability to use

\footnotetext{
${ }^{1}$ For a more detailed account of the functions and evolution of dance throughout history, the interested reader is referred to works by Dils and Albright (2001) and Jonas (1998).

${ }^{2}$ We fully acknowledge that the expression of dance is by no means limited to the human body. Convincing representations of dance can be seen in hand-drawn and computer-generated animation films, marionettes, Sony's Asimo robots, and even stop-motion films of a break-dancing Lego action figure, such as those we are currently working with in our laboratory. Moreover, a cursory look on YouTube provides ample evidence that a veritable menagerie of non-human species also "dances," ranging from cats and dogs to cockatoos and honeybees. A critical evaluation at what actually qualifies as dance is beyond the scope of this paper, and here we focus on intentional dance performed by human agents.
} 
perceptual information to shape movement began in the mid-twentieth century, during which time information processing explanations proposing complex transformations from perception to the organization and execution of action gathered momentum (Massaro and Friedman 1990; Mountcastle et al. 1975; Welford 1968). This line of inquiry received a boost in investigational power with the arrival of advanced neuroimaging techniques in the early 1990s. These tools facilitated the frequency and ease with which scientists could capture a glimpse of the neural architecture and coordinated patterns of activity that support thought, reasoning, action, and emotion.

These techniques enable in vivo investigations of brain function in healthy human subjects through measuring changes in blood flow (including functional magnetic resonance imaging (fMRI), positron emission tomography (PET), and near-infrared spectroscopy) or changes in local electric (electroencephalography) or magnetic (magnetoencephalography) fields beneath the scalp. Our understanding of the relationship between brain structure and function is further advanced by complementary structural neuroimaging methods which enable us to gain an in-depth look at anatomical adaptation in the brain as a consequence of intensive training procedures or the vast quantity of experience in a particular domain seen in experts (Hanggi et al. 2010; Jancke 2009; Scholz et al. 2009). Structural neuroimaging techniques, based on MRI, can provide information both about gray matter features, by means of voxel- or surface-based morphometry, and white matter connectivity, by using diffusion tensor imaging to measure the diffusion properties along fiber tracts. Since the introduction of such techniques, scientists from an ever-expanding range of disciplines including psychology, neuroscience, philosophy, economics, sport science, and now dance are able to explore questions of complex interactions between individuals and their environments in greater detail than ever before, such as the relationship between action production and perception. In this section, we explore how neuroimaging tools are helping to illuminate in new and exciting ways how the brain perceives dance and how an individual's past motor experience sculpts this perception.

Of particular interest to scientists engaged in the study of action is the brain's ability to integrate different types of physical and perceptual experience to learn new movements. Not surprisingly, such abilities to learn new movements are quite pronounced in dancers whose livelihood depends on rapid and adept movement production and reproduction (Stevens et al. 2010). Neuroscientific investigations reveal that a high degree of plasticity within and between seemingly disparate cortical regions and subcortical nuclei gives rise to such movements. Different components of this network of brain regions work together to process action information, both during action perception and during action execution (for comprehensive reviews, see Grafton et al. 2009; Rizzolatti et al. 2009).

Neurophysiologists found the first evidence of a neural system that matches action with perception within the brains of non-human primates. Scientists came across this finding almost by accident, when they were recording from single neurons within the ventral premotor cortex of the monkey brain to determine how these neurons responded when monkeys grasped different items. These researchers observed, much to their surprise, that the same neurons that fired when monkeys performed a specific action (e.g., grasping a raisin) also fired when the monkey 
watched another monkey or a researcher execute the same action (e.g., Rizzolatti et al. 1996a). Subsequent research revealed that these particular neurons do indeed respond preferentially to actions that are either observed or performed, which led researchers to name them "mirror" neurons. As such, mirror neurons appear to compose a cortical network that matches observation of actions with execution of those same actions (Grafton et al. 1996; Rizzolatti et al. 1996b 1995; but see Hagendoorn 2010; Hickok 2009; Turella et al. 2009 for critical counterperspectives to the mirror neuron literature). These specialized neurons have prompted researchers to propose that action perception and production processes form a bidirectional, interactive loop within the primate brain. Since the discovery of mirror neurons in monkeys, many studies have investigated similar functional regions within the human brain, providing evidence for a human action observation network (AON; see Grafton and Hamilton 2007). In addition to the premotor cortex and the inferior parietal lobule (the core mirror system regions discovered in non-human primates; Rizzolatti and Craighero 2004), the action observation network includes the superior temporal sulcus, a region involved in biological motion processing (Blake and Shiffrar 2007), as well as the supplemental motor area, a region implicated in action sequencing and planning (Cross et al. 2009a; Grèzes and Decety 2001).

By now, a rich literature has developed demonstrating a substantial overlap between action perception, performance, and even imagery with techniques that measure corticospinal excitability (Fadiga et al. 1995, 1999, 2005) and changes in blood oxygenation level in motor areas of the brain with fMRI (Grafton et al. 1996, 2009; Rizzolatti and Craighero 2004). Most of these studies focus exclusively on constrained movements of the fingers or hands since such small movements are experimentally easier to study (and also introduce less noise into fMRI recordings) than movements that involve multiple effectors or the whole body. However, some studies have broadened their scope to investigate how these processes interact when viewing, performing, or imagining movements of the feet, legs, hands and feet together, or even the whole body, thus demonstrating the feasibility of investigating perception (and sometimes performance) of more ecologically valid actions where more of the body is observed or used in action (Brown et al. 2006; Catmur et al. 2008).

Several laboratories have turned to populations of expert and novice dancers to further address questions of whole body action cognition. As a recent compendium of work on the subject demonstrates, there are myriad reasons why scientists from the brain and behavioral sciences who are interested in questions of action performance and perception might choose to study dance or dancers (Bläsing et al. 2010). Dance requires a great degree of coordination not only between the different limbs of the body but also between the individual and fellow dancers, perception and action, and time and space. As an example, most dancers can relate to the experience of showing up to a technique class in a new studio, progressing with ease through the warm up or barre exercises, and then being expected to perfectly perform long and complex sequences of steps that have been rapidly demonstrated in the most cursory manner. Dancers' ability to transform scant visual or verbal information into highly sophisticated and expertly executed movements has great potential value to scientists. Significant utility exists in examining both novice and highly experienced 
dancers to see how complex movements are learned, remembered, and reproduced. The study of dance improvisation provides another fruitful paradigm for investigating action production as the brain works to harness properties of the motor system with principles of aesthetic experience to create movements with no predetermined motor program (Hagendoorn 2003). Not only can scientists learn about the coordination and expression of complex actions by quantifying dancers' behavioral performance, but also careful measurement of how such skilled actions are represented at the neural level can shed additional light on how the human body is capable of learning and performing such complex movements with limited information.

Elegant work using dancers and dance-related experimental paradigms to investigate the brain's negotiation between action and perception continues to emerge from an increasing number of functional and structural neuroimaging laboratories (Brown et al. 2006; Calvo-Merino et al. 2005, 2006; Cross et al. 2006, 2009a, b; Hanggi et al. 2010; Orgs et al. 2008). ${ }^{3}$ One of the first of these studies investigated how prior dance experience influences perception of different styles of dance movement (Calvo-Merino et al. 2005). For this experiment, the authors asked male professional ballet dancers, professional capoerira dancers, and age-matched non-dancer control subjects to watch short video clips of a ballet dancer or capoeira dancer performing movements from their respective movement vocabularies. The authors demonstrated experience-specific responses within parietal and premotor components of the AON such that viewing one's own movement vocabulary resulted in stronger activation than viewing the movement vocabulary from a dance style with which one lacks expertise. The authors conclude that an individual's motor repertoire significantly sculpts responses within sensorimotor regions of the brain during action execution.

Cross and colleagues performed a related study on newly acquired action expertise in modern dancers (Cross et al. 2006). In this study, they followed a company of modern dancers as they learned the intricate and complex choreography to Skylight (Dean 1982), a demanding 25-min modern dance work. After each week of rehearsal, the authors scanned the dancers' brains while they watched Skylight and similar but unrehearsed choreography and asked the dancers to rate their current ability to perform each dance segment. Cross et al. (2006) report that as the dancers transitioned from being inexperienced with performing the work to being highly adept performers, neural responses increased significantly and focally in two core components of the AON, namely the inferior parietal lobule and the ventral premotor cortex within the left hemisphere. This study thus demonstrates that changes in sensorimotor cortex were seen while observing dance track with actual physical ability to perform the movements, and these changes in the brain do not require years of rehearsal to manifest - a mere 6 weeks of rehearsal is enough to see marked changes in neural response profiles. These researchers have further explored the effects of de novo dance learning in populations of novice dancers, demonstrating

\footnotetext{
${ }^{3}$ It must be noted, however, that each of these studies represents a small step toward understanding how dance is perceived, represented, and expressed through the brain. It is not yet possible to measure brain activity while dancers are upright, weight-bearing, and moving through space as they might do on stage, in the club, or at the studio. Bearing this fact in mind, we can still use functional neuroimaging techniques to begin to understand the relationship between observing and moving the body in dance contexts.
} 
the utility of dance learning paradigms to address questions of observational learning (Cross et al. 2009b) and how different cues for learning dance are represented within the AON (Cross et al. 2009a).

In another highly innovative study, Brown and colleagues used PET to investigate the core elements of tango dancing (entrainment, meter, and step patterns) by having participants physically perform the foot patterns on an inclined surface while lying in the scanner (Brown et al. 2006). Brown and colleagues report activation within a broad network of mirror system regions when executing complex foot sequences and involvement of striatal and cerebellar components when more complex rhythmic sequences were performed. The activation of these brain regions is consistent with those regions activated by dance observation (Calvo-Merino et al. 2005) and dance imagery paired with observation (Cross et al. 2006). ${ }^{4}$

The research on dancers' brains has opened a portal to understanding how specific changes in the brain are associated with individuals' abilities to perform highly complex and coordinated actions. However, the findings discussed above stop short at being able to explain how and why the observer, or even the dancer, derives such pleasure from the art of dance. In the next section, we introduce the nascent field of neuroaesthetics, a discipline whose express purpose is to use the tools and paradigms discussed above to address questions about which features of artistic expression the brain responds to. This line of inquiry could prove to be particularly fruitful as it has the potential to not only inform scientists about how the brain beholds and appreciates art but could also provide some direct benefits back to the dance community, as we explore below.

\section{Part II: Neuroaesthetics: a neuroscientific portrait of art}

Moving beyond the relative objectivity of neuroscience and into the realm of subjective experience, we discover many intriguing questions upon which we might train our scientific lens. Some of these questions concern why art is of such extreme importance to our senses and why we dedicate such enormous financial resources and time toward the creation and enjoyment of artworks. Across a diverse range of manifestations - including music, dance, and painting - art has been developed by the human species since its early dawn and continues to endure as a critical presence across all cultures (Mithen 1996). Over millennia, the synergistic coexistence of humans and art has fed an extensive and rich debate on the function of art and the essence of aesthetic experience. This debate has engaged numerous artists, historians, and philosophers, and now, neuroscientists are getting involved as well. The recent advances in neuroimaging techniques introduced in the previous section, as well as neurostimulation technology (such as transcranial magnetic stimulation), enable us to investigate from "the inside" the cerebral phenomena associated with

\footnotetext{
${ }^{4}$ It is of note that a recent neuromorphometry study of expert ballet dancers has demonstrated an altered morphometry of several of these same neural regions (Hanggi et al. 2009). Such a finding begs the question of whether experience is sculpting the brain to respond when observing dance in such a manner or whether individuals with "dance-disposed" brains are more likely to train to be dancers. Such questions are certainly worthy of further investigation.
} 
artistic creativity and aesthetic appreciation (Fairhall and Ishai 2008; Ishai et al. 2007; Kawabata and Zeki 2004).

Art, like everything we do, is generated from electrical impulses passed between synapses in the brain, expressed through the body, and eventually appreciated through the senses. Therefore, in order to better understand how we perceive and evaluate art, we might gain valuable insights by taking an in-depth look at how stimuli are conveyed from the external world to the brain and how they are transformed into vivid perceptions. So far, fMRI enables us to visualize patterns of activity across different brain areas while we carry out an action, think, or feel a particular emotion. Neuroimaging work has revealed a certain extent of functional localization where individual cerebral structures are specialized for one or more specific tasks, like the elaboration of sensory stimuli (visual, tactile, auditory, etc.), the planning and execution of motor processes, or processing of emotional responses to emotionally valenced stimuli.

Where aesthetics and personal preference are concerned, the story becomes more complicated. If we were to behold a portrait by Vincent Van Gogh, a song played by Miles Davis, or the dance company Pilobolus in action, our individual aesthetic experiences of these works would share commonalities, as well as striking differences. Feelings, memories, and perceived pleasure likely have a strong individual character because they are attached to genetic, environmental, and cultural components. Such inter-individual variability can even be seen in functional neuroimaging studies of basic emotional responses which reveal marked differences in patterns of neural activity between individuals in the same emotional states (Davidson and Irwin 1999; Eugene et al. 2003). Nonetheless, neuroimaging work on viewing artworks such as film and paintings demonstrates reliable and consistent patterns of neural activation across individuals when we share common experiences, such as watching an exciting scene in a film or beholding a particular painting (e.g., Hasson et al. 2004; Kawabata and Zeki 2004). Shared experiences across individuals, such as may be induced by perceiving an artistic work, have the potential to put us on the same interpretive plane as our fellow spectators. We speculate that shared activations during shared experiences might be one of the reasons why we can communicate - through art-impressions and emotions that might not be possible to express through words. What is clear so far is that more indepth inquiry into the aesthetic evaluation of artworks should turn toward investigation of the underlying physical properties and changes within the brain as it is solely this organ that is responsible for generating and evaluating aesthetic experience. For such reasons, in recent years, philosophers, physicists, engineers, and biologists who study the brain have increasingly been interested to a scientific approach to the study of aesthetics in a number of domains, giving rise to the emerging field of neuroaesthetics (e.g., Fritz et al. 2009; Jacobsen et al. 2006; Koelsch 2010; Limb and Braun 2008; Nadal et al. 2008; Zaidel 2005; Zatorre et al. 2007; Zeki 2000).

Although it is sometimes dismissed as reductionism, neuroaesthetics is not seeking to demonstrate a universal formula of aesthetic appreciation or of creativity that would overcome the complexity of art and at least 2,000 years of aesthetic studies. As a matter of fact, we cannot accept, to quote Oscar Wilde, "any theory of beauty in exchange for beauty itself, and, so far from desiring to isolate it in a 
formula appealing to the intellect, we, on the contrary, seek to materialize it in a form that gives joy to the soul through the senses. We want to create it, not to define it" (Wilde 1942). To this end, the aim of neuroaesthetic inquiry should not be the definition of rules or criteria for what makes a work of art successful or beautiful but could instead be to provide artists with an understanding of how their work alters or impacts the neurophysiology of the beholder. Indeed, during past centuries, writers and philosophers including Plato, Immanuel Kant, and art historian Johann Joachim Winckelmann have tried to grasp the intimate essence of the beholder's aesthetic experience and to define the concept of aesthetic judgment. Still, these important figures of Western thought never had the opportunity to directly see what happens in our brains when, for example, we experience a work of art.

Just as fMRI is facilitating research into how we learn and perceive dance, we can use the same technology to begin to investigate the neural underpinnings of aesthetic perception (keeping in mind, however, the significant limitations of an experimental setup where participants lie in a scanner and perceive reproductions of artistic works on a computer screen). The first functional neuroimaging studies on aesthetic perception investigated perception and evaluation of paintings. These experiments demonstrated common engagement of prefrontal cortices when subjects viewed aesthetically pleasing paintings, as opposed to paintings they disliked, regardless of the category of painting (Cela-Conde et al. 2004; Kawabata and Zeki 2004; Vartanian and Goel 2004). More recent work has demonstrated that activity within these areas is significantly biased by subjects' prior expectations about the hedonic value of stimuli (Kirk et al. 2009). These authors demonstrate how a number of external factors can bias brain activation while viewing art works, which was then associated with less impartial aesthetic judgments. The fact that external influences can bias brain activity and consequent aesthetic judgments might offer a scientific explanation as to why we may come to reevaluate more positively a work of art we initially disliked (for example, when we realize who the artist is and that the artist is universally respected or when a work of art is located in a renowned art gallery, cf. Kirk et al. 2009).

Over the past decade, an ever-increasing number of scientific works are providing us a new and privileged point of view of how art perception influences the brain (see (Chatterjee 2011; Di Dio and Gallese 2009). Equally importantly, the cross-fertilization between science and art has fueled an interest to study other forms of human artistic expression beyond painting and sculpture, including dance (Calvo-Merino et al. 2008, 2010; Di Dio and Gallese 2009; Hagendoorn 2004; Cross et al., It is very beautiful, but I cannot do it: The relationship between aesthetic evaluation and action experience in sensorimotor cortices, manuscript in preparation). Dance provides particular challenges and opportunities for the field of neuroaesthetics as it is neither represented by static pictures nor is its perception and evaluation primarily restricted to the visual system. Experiments aiming to investigate affective evaluations of dance make use of dynamic visual displays of a body or bodies moving through space, sometimes with the addition of music. As discussed in the previous section, observation of dance activates much more than just visual regions of the brain, including premotor and parietal cortices (CalvoMerino 2010; Cross 2010). The first neuroimaging study to investigate aesthetic responses to dance has demonstrated the active engagement of sensorimotor brain areas when observing dance movements that one finds to be aesthetically pleasing 
compared to those judged to be less pleasing, thus suggesting a possible role for the motor system in dance appreciation (Calvo-Merino et al. 2008). The complex and scenographic use of human bodies evokes visceromotor and somatomotor resonance, in addition to activating emotional and reward-related centers within the brain (Cinzia and Vittorio 2009). Recent research seems to suggest that multisensory perceptions are generally preferred to perceptions in just one of the sensory domains (Ward et al. 2008). To this end, dance might be a particularly good candidate art form for investigating more complex questions of aesthetic appreciation as watching dance evokes sensation across the visual and sensorimotor domains (and the auditory domain as well, if music or sound is involved).

Although the study of dance neuroaesthetics is only just beginning, it is a field ripe with possibilities for future work, which could be of substantial benefit to individuals in the dance community as well as the brain sciences. As discussed by Hagendoorn (2010), literally millions of dance videos can be viewed on YouTube, some of which boast over 200 million views. These videos range from the polished productions of Lady Gaga and the Nederlands Dans Theatre to low-fidelity home videos of dancing babies or people bursting into dance at a Belgian train station. One possibility for future work to explore is whether when people watch different styles and forms of dance common patterns of brain activation emerge when viewing aesthetically pleasing dance, independent of dance style or staging context. Work in this vein has the potential to inform not only scientists engaged in neuroaethetic studies but also choreographers. For example, findings from such investigations could inspire innovative new dance works where notions of style (i.e., contemporary dance, break dance, classical ballet, square dance) and context (costumes, lighting, stage, etc.) are blended or interchanged in innovative new ways that neuroimaging data suggest might be successful. Similar ideas about using psychological and neurophysiological data to inform the choreographic process are discussed at length by Hagendoorn $(2003,2010)$. Another question dance neuroaesthetics might address that would be of interest to the dance community concerns what differences are manifest within the brains of dance aficionados and those who attend dance performances only when they are dragged to them against their will by an enthusiastic friend, family member, or partner. Are some people's brains just not responsive to watching dance at all? How might this be related to one's desire to dance oneself? Is dislike of dance a stable neurophysiological trait, or might different interventions change people's responses to dance, both at affective and neural levels? Exploring such questions will take the first steps to address the challenge to answer bigger questions about why we enjoy dance, such as what it is about dance, precisely, that makes us want to watch or perform it ourselves.

\section{Conclusion}

Long ago, Hippocrates understood how perceptions, feelings, and creativity were intimately correlated to the mind, but even more so to its most tangible physical element, the brain. Since accruing evidence from the brain sciences lends support to this assumption, every form of art, dance included, has the potential to be better understood through investigating how the brain responds when perceiving art. We 
are convinced that an increased understanding of expert and novice dancers' brains, as well as the patterns of neural activation observed in dance spectators' brains while watching dance, will not only help unravel how the brain learns and represents complex actions but might also help us understand why the arts have such importance in our society. Exploration of the neural mechanisms associated with art appreciation, such as the biological reasons why certain works or performances are more popular than others, is undoubtedly of considerable artistic and commercial value (Ariely and Berns 2010). Vice versa, studying the nature of aesthetic appreciation can also help us to better understand the mechanisms underlying perception and the strategies our brains use to process the world around us. In the 10 years since Zeki's seminal publication of Inner Vision, the number of neuroaesthetic studies has multiplied exponentially, and the field continues to gain momentum. However, as Chatterjee (2011) warns, it is critical that future studies in the neuroaesthetic domain should make clear what, precisely, neuroscientific data add to the study of aesthetics that behavioral experiments alone cannot achieve. We strongly believe that this caution should be taken seriously by future studies investigating the neuroaesthetics of dance. If it is, future work on how dance learning and observation interact with aesthetic experience has the potential to advance our understanding of dance creation and appreciation.

Acknowledgments We thank Rick Ramsey and the anonymous reviewers for helpful comments on a previous version of the manuscript. EC acknowledges support from a postdoctoral research fellowship from the Alexander von Humboldt Foundation.

Open Access This article is distributed under the terms of the Creative Commons Attribution Noncommercial License which permits any noncommercial use, distribution, and reproduction in any medium, provided the original author(s) and source are credited.

\section{References}

Ariely, D., \& Berns, G. S. (2010). Neuromarketing: The hope and hype of neuroimaging in business. Nature Reviews Neuroscience, 11(4), 284-292.

Blake, R., \& Shiffrar, M. (2007). Perception of human motion. Annual Review of Psychology, 58, 47-73.

Bläsing, B., Puttke, M., \& Schack, T. (Eds.). (2010). The neurocognition of dance. London: Psychology Press.

Boyer, P., \& Lienard, P. (2006). Why ritualized behavior? Precaution Systems and action parsing in developmental, pathological and cultural rituals. The Behavioral and Brain Sciences, 29(6), 595-613. discussion 613-550.

Brown, S., Martinez, M. J., \& Parsons, L. M. (2006). The neural basis of human dance. Cerebral Cortex, 16(8), 1157-1167.

Calvo-Merino, B. (2010). Neural mechanisms for seeing dance. In B. Bläsing, M. Puttke, \& T. Schack (Eds.), The neurocognition of dance. London: Psychology Press.

Calvo-Merino, B., Glaser, D. E., Grèzes, J., Passingham, R. E., \& Haggard, P. (2005). Action observation and acquired motor skills: An FMRI study with expert dancers. Cerebral Cortex, 15(8), 1243-1249.

Calvo-Merino, B., Grèzes, J., Glaser, D. E., Passingham, R. E., \& Haggard, P. (2006). Seeing or doing? Influence of visual and motor familiarity in action observation. Current Biology, 16(19), 1905-1910.

Calvo-Merino, B., Jola, C., Glaser, D. E., \& Haggard, P. (2008). Towards a sensorimotor aesthetics of performing art. Consciousness and Cognition, 17(3), 911-922.

Calvo-Merino, B., Urgesi, C., Orgs, G., Aglioti, S. M., \& Haggard, P. (2010). Extrastriate body area underlies aesthetic evaluation of body stimuli. Experimental Brain Research, 204(3), 447-456. 
Catmur, C., Gillmeister, H., Bird, G., Liepelt, R., Brass, M., \& Heyes, C. (2008). Through the looking glass: Counter-mirror activation following incompatible sensorimotor learning. European Journal of Neuroscience, 28, 1208-1215.

Cela-Conde, C. J., Marty, G., Maestu, F., Ortiz, T., Munar, E., Fernandez, A., et al. (2004). Activation of the prefrontal cortex in the human visual aesthetic perception. Proceedings of the National Academy of Sciences of the United States of America, 101(16), 6321-6325.

Chatterjee, A. (2011). Neuroaesthetics: A coming of age story. Journal of Cognitive Neuroscience, 23, 53-62.

Cinzia, D. D., \& Vittorio, G. (2009). Neuroaesthetics: A review. Current Opinion in Neurobiology, 19, $682-687$.

Cross, E. S. (2010). Building a dance in the human brain: Insights from expert and novice dancers. In B. Bläsing, M. Puttke, \& T. Schack (Eds.), The neurocognition of dance. London: Psychology Press.

Cross, E. S., Hamilton, A. F., \& Grafton, S. T. (2006). Building a motor simulation de novo: Observation of dance by dancers. Neuroimage, 31(3), 1257-1267.

Cross, E. S., Hamilton, A. F., Kraemer, D. J., Kelley, W. M., \& Grafton, S. T. (2009a). Dissociable substrates for body motion and physical experience in the human action observation network. The European Journal of Neuroscience, 30(7), 1383-1392.

Cross, E. S., Kraemer, D. J., Hamilton, A. F., Kelley, W. M., \& Grafton, S. T. (2009b). Sensitivity of the action observation network to physical and observational learning. Cerebral Cortex, 19(2), 315-326.

Davidson, R. J., \& Irwin, W. (1999). The functional neuroanatomy of emotion and affective style. Trends in Cognitive Sciences, 3(1), 11-21.

Dean, L. (Artist) (1982). Skylight [Dance].

Di Dio, C., \& Gallese, V. (2009). Neuroaesthetics: A review. Current Opinion in Neurobiology, 19, $682-687$.

Dils, A., \& Albright, A. C. (2001). Moving history/dancing cultures: A dance history reader. Middletown: Wesleyan University Press.

Eugene, F., Levesque, J., Mensour, B., Leroux, J. M., Beaudoin, G., Bourgouin, P., et al. (2003). The impact of individual differences on the neural circuitry underlying sadness. Neuroimage, 19 (2 Pt 1), 354-364.

Fadiga, L., Fogassi, L., Pavesi, G., \& Rizzolatti, G. (1995). Motor facilitation during action observation: A magnetic stimulation study. Journal of Neurophysiology, 73(6), 2608-2611.

Fadiga, L., Buccino, G., Craighero, L., Fogassi, L., Gallese, V., \& Pavesi, G. (1999). Corticospinal excitability is specifically modulated by motor imagery: A magnetic stimulation study. Neuropsychologia, 37(2), 147-158.

Fadiga, L., Craighero, L., \& Olivier, E. (2005). Human motor cortex excitability during the perception of others' action. Current Opinion in Neurobiology, 15(2), 213-218.

Fairhall, S. L., \& Ishai, A. (2008). Neural correlates of object indeterminacy in art compositions. Consciousness and Cognition, 17(3), 923-932.

Fritz, T., Jentschke, S., Gosselin, N., Sammler, D., Peretz, I., Turner, R., et al. (2009). Universal recognition of three basic emotions in music. Current Biology, 19(7), 573-576.

Grafton, S. T., Arbib, M. A., Fadiga, L., \& Rizzolatti, G. (1996). Localization of grasp representations in humans by positron emission tomography. 2. Observation compared with imagination. Experimental Brain Research, 112(1), 103-111.

Grafton, S. T., Aziz-Zadeh, L., \& Ivry, R. B. (2009). Relative hierarchies and the representation of action. In M. S. Gazzaniga (Ed.), The cognitive neurosciences (4th ed., pp. 641-654). Boston: MIT Press.

Grafton, S. T., \& Hamilton, A. F. (2007). Evidence for a distributed hierarchy of action representation in the brain. Human Movement Science, 26(4), 590-616.

Grèzes, J., \& Decety, J. (2001). Functional anatomy of execution, mental simulation, observation, and verb generation of actions: A meta-analysis. Human Brain Mapping, 12(1), 1-19.

Hagendoorn, I. G. (2003). Cognitive dance improvisation: How study of the motor system can inspire dance (and vice versa). Leonardo, 36(3), 221-227.

Hagendoorn, I. G. (2004). Some speculative hypotheses about the nature and perception of dance and choreography. Journal of Consciousness Studies, 11(3/4), 79-110.

Hagendoorn, I. G. (2010). Dance, choreography, and the brain. In D. Melcher \& F. Bacci (Eds.), Art and the senses (pp. 499-514). Oxford: Oxford University Press.

Hanggi, J., Koeneke, S., Bezzola, L., \& Jancke, L. (2010). Structural neuroplasticity in the sensorimotor network of professional female ballet dancers. Human Brain Mapping, 31, 1196-1206.

Hasson, U., Nir, Y., Levy, I., Fuhrmann, G., \& Malach, R. (2004). Intersubject synchronization of cortical activity during natural vision. Science, 303(5664), 1634-1640.

Hickok, G. (2009). Eight problems for the mirror neuron theory of action understanding in monkeys and humans. Journal of Cognitive Neuroscience, 21(7), 1229-1243. 
Ishai, A., Fairhall, S. L., \& Pepperell, R. (2007). Perception, memory and aesthetics of indeterminate art. Brain Research Bulletin, 73(4-6), 319-324.

Jacobsen, T., Schubotz, R. I., Hofel, L., \& Cramon, D. Y. (2006). Brain correlates of aesthetic judgment of beauty. Neuroimage, 29(1), 276-285.

James, W. (1890). Principals of psychology. New York: Holt.

Jancke, L. (2009). The plastic human brain. Restorative Neurology and Neuroscience, 27(5), 521-538.

Jonas, G. (1998). Dancing: The pleasure, power, and art of movement. New York: Harry N. Abrams.

Kawabata, H., \& Zeki, S. (2004). Neural correlates of beauty. Journal of Neurophysiology, 91(4), 16991705.

Kirk, U., Skov, M., Christensen, M. S., \& Nygaard, N. (2009). Brain correlates of aesthetic expertise: A parametric fMRI study. Brain and Cognition, 69(2), 306-315.

Koelsch, S. (2010). Towards a neural basis of music-evoked emotions. Trends in Cognitive Sciences, 14 (3), 131-137.

Limb, C. J., \& Braun, A. R. (2008). Neural substrates of spontaneous musical performance: An FMRI study of jazz improvisation. PLoS ONE, 3(2), e1679.

Massaro, D. W., \& Friedman, D. (1990). Models of integration given multiple sources of information. Psychological Review, 97(2), 225-252.

Mithen, S. J. (1996). The prehistory of the mind: A search for the origns of art, religion, and science. London: Thames and Hudson.

Mountcastle, V. B., Lynch, J. C., Georgopoulos, A., Sakata, H., \& Acuna, C. (1975). Posterior parietal association cortex of the monkey: Command functions for operations within extrapersonal space. Journal of Neurophysiology, 38(4), 871-908.

Nadal, M., Munar, E., Capo, M. A., Rossello, J., \& Cela-Conde, C. J. (2008). Towards a framework for the study of the neural correlates of aesthetic preference. Spatial Vision, 21(3-5), 379-396.

Orgs, G., Dombrowski, J. H., Heil, M., \& Jansen-Osmann, P. (2008). Expertise in dance modulates alpha/beta event-related desynchronization during action observation. The European Journal of Neuroscience, 27 (12), 3380-3384.

Rizzolatti, G., \& Craighero, L. (2004). The mirror-neuron system. Annual Review of Neuroscience, 27, $169-192$.

Rizzolatti, G., Fadiga, L., Gallese, V., \& Fogassi, L. (1996a). Premotor cortex and the recognition of motor actions. Cognitive Brain Research, 3(2), 131-141.

Rizzolatti, G., Fadiga, L., Matelli, M., Bettinardi, V., Paulesu, E., Perani, D., et al. (1996b). Localization of grasp representations in humans by PET: 1. Observation versus execution. Experimental Brain Reseach, 111(2), 246-252.

Rizzolatti, G., Fogassi, L., \& Gallese, V. (2009). The mirror neuron system. In M. S. Gazzaniga (Ed.), The cognitive neurosciences (4th ed., pp. 625-640). Boston: MIT Press.

Scholz, J., Klein, M. C., Behrens, T. E., \& Johansen-Berg, H. (2009). Training induces changes in white-matter architecture. Nature Neuroscience, 12(11), 1370-1371.

Stevens, C., Winskel, H., Howell, C., Vidal, L. M., Latimer, C., \& Milne-Home, J. (2010). Perceiving dance: Schematic expectations guide experts' scanning of a contemporary dance film. Journal of Dance Medicine \& Science, 14(1), 19-25.

Turella, L., Pierno, A. C., Tubaldi, F., \& Castiello, U. (2009). Mirror neurons in humans: Consisting or confounding evidence? Brain and Language, 108(1), 10-21.

Vartanian, O., \& Goel, V. (2004). Neuroanatomical correlates of aesthetic preference for paintings. NeuroReport, 15(5), 893-897.

Ward, J., Moore, S., Thompson-Lake, D., Salih, S., \& Beck, B. (2008). The aesthetic appeal of auditory-visual synaesthetic perceptions in people without synaesthesia. Perception, 37(8), 1285-1296.

Welford, A. T. (1968). Fundamentals of skill. London: Methuen.

Wilde, O. (1942). Lecture to art students The Complete Works of Oscar Wilde. London: Wordsworth Editions Limited.

Zaidel, D. W. (2005). Neuropsychology of art: Neurological, cognitive and evolutionary perspectives. East Sussex: Psychology Press.

Zatorre, R. J., Chen, J. L., \& Penhune, V. B. (2007). When the brain plays music: Auditory-motor interactions in music perception and production. Nature Reviews. Neuroscience, 8(7), 547-558.

Zeki, S. (2000). Inner vision: An exploration of art and the brain. Oxford: Oxford University Press. 